\section{Die Stärken der Direkten Medikamentenabgabe}

\author{
Die Direkte Medikamentenabgabe (DMA) ist wohl \\ neben intelligent aufgebauten Hausarztmodellen die \\ kosteneffizienteste Einrichtung des Schweizerischen \\ Gesundheitswesens. Diese DMA gilt es zu bewahren \\ und zu fördern, erst recht im neuen Jahr und entge- \\ gen allen Unkenrufen, die nach deren Abschaffung \\ infolge "wirklicher Bedrohung der Apothekenexi- \\ stenz" schreien. \\ Es geht, wie die Abstimmung im Kanton Zürich \\ gezeigt hat, um die Wahlfreiheit der Patienten und \\ nicht um eine Strukturerhaltung in einem über- \\ regulierten Gesundheitswesen, in welchem je nach \\ Interesse nach mehr Markt oder nach mehr Sicher- \\ heit für die Patienten - im Klartext: noch mehr \\ Regulierung - geschrien wird.
}

\section{Avantages de la propharmacie}

La propharmacie ou distribution directe de médicaments par le médecin représente, hormis les modèles de médecin de famille intelligemment conçus, le système le plus efficace en matière de coûts dans notre système de santé suisse. Il s'agit donc de sauvegarder, d'encourager la propharmacie, précisément en ce début d'année nouvelle. Eloignons les Cassandre qui réclament $\dot{a}$ cor et $\dot{a}$ cri son abolition $d u$ fait qu'elle "menace l'existence des pharmacies".

Le vote cantonal zurichois nous l'a montré, il s'agit de garantir la liberté de choix du patient et non de préserver la structure d'un système sanitaire surréglementé qui, suivant les intérêts en présence, prône la toute-puissance du marché ou la primauté sécuritaire pour les patients, donc davantage de contraintes.
Die Ärzte mit DMA setzen eine ihnen wohlbekannte und bewusst auf ihre Praxistätigkeit zugeschnittene Palette von Arzneimitteln ein. Diese Arzneimittel sind für alle Notfälle griffbereit. Gefährliche Interaktionen können so vermieden werden. Durch die DMA wird die Compliance gefördert, Pharmamüll wird durch die dem Therapieziel angepasste Arzneimittelmenge verhindert, und Generika werden entsprechend den vom KVG geforderten Kriterien der Wirksamkeit, Zweckmässigkeit und Wirtschaftlichkeit eingesetzt. Zu guter Letzt ist die DMA seit Einführung der neuen Spezialitätenliste (SL) vom 1. Juli 2001, Datum der Einführung des leistungsorientierten Abgeltungsmodells (LOA) für Apotheker, die günstigste Art, SL-pflichtige Arzneimittel abzugeben.

Da bleibt nur noch eine Frage zu beantworten: Warum sträuben sich Politiker und Vertreter von Krankenversicherungen gegen diese kosteneffizienteste Art der Arzneimittelabgabe?

Max Giger, Winterthur

Les médecins pratiquant la propharmacie optent pour une gamme de médicaments qu'ils connaissent bien, qui sont adaptés à leur activité médicale et accessibles pour tous les cas d'urgence. Cette façon de faire permet d'éviter les interactions dangereuses.

La propharmacie encourage également l'adhésion au traitement et évite les excès de déchets médicamenteux, grâce à une remise de médicaments dans des quantités adaptées au but thérapeutique. De plus, la prescription de génériques se fait dans le respect des critères d'efficacité, d'adéquation et de caractère économique exigés par la LAMal. Enfin, depuis l'introduction de la nouvelle liste des spécialités (LS) du $1^{\text {er }}$ juillet 2001, date de l'introduction du modèle de rémunération basée sur les prestations (RBP) pour les pharmaciens, la propharmacie est le mode le plus avantageux de délivrer des médicaments soumis à la LS.

Un mystère demeure: pourquoi le monde politique et les représentants des assurances-maladie combattent avec tant d'énergie un mode de distribution des médicaments aussi rentable?

Max Giger, Winterthur 